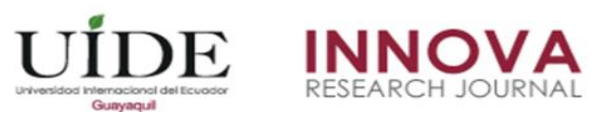

INNOVA Research Journal, ISSN 2477-9024

(Julio, 2017). Vol. 2, No.7 pp. 189-195

DOI: https://doi.org/10.33890/innova.v2.n7.2017.335

URL: http://revistas.uide.edu.ec/index.php/innova/index

Correo: innova@uide.edu.ec

\title{
Análisis comparativo de la concentración de BTX en talleres automotrices
}

\section{Comparative analysis of the concentration of BTX in automotive workshops}

Juan Fernando Iñiguez

Gorky Reyes

Marco Cruz

Diego Pérez

Universidad Internacional del Ecuador, Ecuador

Autor por Correspondencia: jiniguez@uide.edu.ec, gureyesca@uide.edu.ec, cgm11027188@ hotmail.com.

Fecha de recepción: 30 de Abril de 2017 - Fecha de aceptación: 30 de Junio de 2017

Resumen: Los disolventes en aerosoles usados para el mantenimiento automotriz emiten componentes volátiles que superan los límites permisibles de componentes orgánicos volátiles en ambientes laborales debido a la estructura abierta o cerrada del ambiente laboral de los talleres automotrices por lo cual es necesario un análisis de concentraciones entre estos dos tipos de ambientes para un mejor entendimiento del comportamiento de los componentes orgánicos volátiles en estos ambientes. Se realizó la toma de muestras de aire durante el uso de aerosoles disolventes para la limpieza de autopartes, limpieza de frenos, limpieza del cuerpo de aceleración y en el ambiente general de los talleres automotrices basándose en el método MTA/MA-030/A92 posteriormente enviadas a un Laboratorio normado para obtener los datos cuantitativos. En los talleres automotrices de ambiente cerrado en las cuatro categorías de mantenimiento se evidencio la presencia de Benceno entre un 0,1030 - 0,4510 mg/m3, Tolueno entre 0,5770 - 1,2220 mg/m3 y Xileno entre 0 - 0,3850 mg/m3 mientras que en el ambiente abierto se evidencio la presencia de Benceno entre $0-0,4900 \mathrm{mg} / \mathrm{m} 3$, Tolueno entre $0-0,4600 \mathrm{mg} / \mathrm{m} 3$ y Xileno entre $0-0,7500 \mathrm{mg} / \mathrm{m} 3$. Los talleres automotrices " $\mathrm{A}$ " $\mathrm{y}$ " $\mathrm{B}$ " poseen mayores concentraciones de Benceno con un promedio de $0,3770 \mathrm{mg} / \mathrm{m} 3$, Tolueno con $1,1885 \mathrm{mg} / \mathrm{m} 3$ y Xileno con $0,3515 \mathrm{mg} / \mathrm{m} 3$ durante la utilización de aerosoles para la limpieza de autopartes y frenos, sin embargo, estos niveles de concentración no superan los límites permisibles mientras que el taller automotriz " $C$ " posee una menor incidencia de componentes volátiles debido a un mayor flujo de aire.

Palabras clave: COVs, concentración; adsorción; carbón activo; ventilación natural Abstract: Aerosol solvents used for automotive maintenance emit volatile components that can exceed permissible limits due to the open or closed structure of the work environment of automotive workshops, so a concentration analysis between these two types of environments is necessary. Air samples were taken during the use of solvent aerosols for cleaning of auto parts, cleaning of brakes, cleaning of the acceleration body and in the general atmosphere of the automotive workshops "A", "B" and "C" "Based on the MTA / MA-030 / A92 method subsequently sent to a standardized laboratory to obtain quantitative data. The presence of Benzene between 0.1030 and $0.4510 \mathrm{mg} / \mathrm{m} 3$ was observed in the "A" and "B" automotive workshops in the four maintenance categories, Toluene between $0.5770-1.2220 \mathrm{mg} / \mathrm{m} 3$ and Xylene between $0-0.3850 \mathrm{mg} / \mathrm{m} 3$ and in the automotive workshop " $\mathrm{C}$ " the presence of Benzene between 0- 
$0.4900 \mathrm{mg} / \mathrm{m} 3$, Toluene between 0 - $0.4600 \mathrm{mg} / \mathrm{m} 3$ and Xylene between 0 and $0.3850 \mathrm{mg} /$ $0.7500 \mathrm{mg} / \mathrm{m}$ 3.Automotive workshops "A" and "B" have higher concentrations of Benzene with an average of $0.3770 \mathrm{mg} / \mathrm{m} 3$, Toluene with $1.1885 \mathrm{mg} / \mathrm{m} 3$ and Xylene with $0.3515 \mathrm{mg} / \mathrm{m} 3$ during the use of aerosols For the cleaning of auto parts and brakes, however these concentration levels do not exceed the permissible limits while the automotive workshop " $\mathrm{C}$ " has a lower incidence of volatile components due to a greater air flow.

Key words: VOCs; concentration; adsorption; active carbon; natural ventilation

\section{Introducción}

Los automóviles actualmente en la sociedad se han vuelto una necesidad para el transporte por lo que el parque automotor ha ido creciendo continuamente convirtiéndose en una fuente de contaminación para el medio ambiente y los seres humanos. (Krzystowczyk, 2011) El funcionamiento de estos automóviles y su mantenimiento provocan una variedad de contaminantes ambientales. (Weisel, 2010) La pulverización de productos químicos es el medio más eficaz de la aplicación de disolventes, penetradores, lubricantes, y productos de limpieza para el mantenimiento de vehículos, la desventaja de la facilidad de aplicación de estos productos químicos es que se volatilizan en el aire, se inhalan fácilmente en el sistema respiratorio y contienen COVs (Chilton, 2000). Se ha realizado varios estudios de componentes orgánicos volátiles asociados a los riesgos laborales químicos en estaciones de gasolina de España, México, Francia y Grecia, reportando componentes orgánicos volátiles en los ambientes laborares. (Moolla, 2015)

En Canadá se han llevado estudios de componentes orgánicos volátiles en talleres automotrices que han demostrado la presencia de Benceno, Tolueno, Xileno (Badjagbo, 2010)

Los compuestos químicos orgánicos están en todas partes tanto en ambientes internos y externos, ya que se han convertido en componentes químicos esenciales en muchos productos y materiales (Vega, 2010). Mientras que los compuestos orgánicos volátiles (COV) son compuestos de carbono, excepto los designados por la EPA como el monóxido de carbono, dióxido de carbono o carburos metálicos que participan en reacciones fotoquímicas atmosféricas (EPA, 2016).

\section{Riesgos Químicos}

Son aquellos riesgos producidos por una exposición no controlada a agentes químicos. (Authority, 2004) La exposición a los productos químicos causa o contribuir a muchos afectos serios sobre la salud tales como enfermedades del corazón, lesiones a los riñones y a los pulmones, esterilidad, cáncer, quemaduras y erupciones además pueden causar incendios, explosiones y demás accidentes de envergadura (3117).

\section{Ventilación.}

La industria moderna, con las nuevas tecnologías y la variedad de operaciones y procesos que se realiza en las diferentes áreas como por ejemplo en la automotriz se utiliza un número cada vez mayor de compuestos y sustancias químicas, muchas de las cuales son altamente 
nocivas para la salud de los trabajadores. El estrés que se genera debido al calor del ambiente provoca un ambiente laboral inseguro o incómodo (Industrial, 2012).

Las ventilación recomendada para los talleres mecánicos va de $0,25-0,50 \mathrm{~m} / \mathrm{s}$ para mantener un ambiente confortable (colaboradores, 2010).

\section{Normativas}

Por otro lado, las normativas internacionales han establecido límites permisibles de exposición a compuestos orgánicos volátiles (COVs) como son la Administración de Seguridad y Salud ocupacional (OSHA), el REAL DECRETO 374/2001 de España, el Instituto Nacional de Seguridad y Salud Ocupacional (NIOSH) como se describen en la Tabla 1 los "Límites de exposición profesional para agentes químicos 2015" ((INSHT), 2015).

Tabla 1. Límites de Exposición Profesional para Agentes Químicos 2015.

\begin{tabular}{lll}
\hline CAS & Agente Químico & VLA-ED mg/m3 \\
\hline $1330-20-7$ & Xilenos & 221 \\
$108-88-3$ & Tolueno & 192 \\
$71-43-2$ & Benceno & 3.25 \\
\hline
\end{tabular}

Fuente: Límites de exposición profesional para agentes químicos en España 2015; Instituto Nacional de Seguridad e Higiene en el Trabajo (INSHT); Madrid, Enero 2015.

\section{Materiales y métodos}

Se analizaron los talleres automotrices "A", "B" y "C" en cuatro ambientes laborales donde se evidencio una alta frecuencia de uso de aerosoles limpiadores con componentes orgánicos volátiles. Se identificaron 3 productos usados en los talleres automotrices detallando su contenido en la Tabla 2 según las hojas de seguridad (MSDS).

Tabla 2 Contenido de Espray Limpiadores Automotrices

\begin{tabular}{lllllll}
\hline & Acetona & Tolueno & Metanol & Dióxido de carbono & Heptano & Xileno \\
\hline Producto \# 1 & $45-55 \%$ & $25-35 \%$ & $10-20 \%$ & $5-10 \%$ & $0 \%$ & $0 \%$ \\
Producto \# 2 & $40-50 \%$ & $20-30 \%$ & $0 \%$ & $5-15 \%$ & $10-20 \%$ & $0 \%$ \\
Producto \# 3 & $15-40 \%$ & $0 \%$ & $0 \%$ & $0 \%$ & $5-10 \%$ & $5-10 \%$ \\
\hline
\end{tabular}

\section{Fuente: Hoja de datos de seguridad de material}

Se analizó la concentración de COVs mediante la bomba de muestreo personal CASTELLA TUFF IS con la participación de ocho técnicos automotrices en dos talleres automotrices de ambiente cerrado y cuatro técnicos en un taller automotriz de ambiente abierto (Tabla 3).

Tabla 3. Características de los talleres automotrices

\begin{tabular}{cccc}
\hline Taller & $\begin{array}{c}\text { Vol. } \\
\text { inter, m3 }\end{array}$ & $\begin{array}{c}\text { Cap. } \\
\text { vehicular }\end{array}$ & $\begin{array}{c}\text { Técnicos } \\
\text { Analizados }\end{array}$ \\
\hline A & 1861 & 16 & 4 \\
B & 3973 & 18 & 4 \\
\hline
\end{tabular}




\begin{tabular}{llll}
\hline $\mathrm{C}$ & 3964 & 23 & 4 \\
\hline
\end{tabular}

Fuente: Autores

Para la toma de muestras se usaron tubos de carbón activo de $6 \mathrm{~mm}$ de diámetro, tomadas por la mañana en condiciones normales de trabajo durante 6 días. Se analizó cuatro categorías de mantenimiento automotriz más relevantes: Ambiente General, Mantenimiento de frenos, Limpieza del cuerpo de aceleración, Limpieza de autopartes. La toma de muestras se realizó durante 20 minutos en cada categoría con 1 bombas Castella Tuff Is con una capacidad de 0,5 a 4,5 1/min. En la toma de muestras de ambientes generales se realizó a 1 metro de distancia de las bahías de trabajo. En total se obtuvieron 12 muestras de aire. Las muestras fueron enviadas a los laboratorios Himalaya en Málaga, España donde se aplicó el método "Determinación de hidrocarburos, vapores orgánicos y alcoholes en aire por el Método de adsorción en carbón activo (MTA/MA-030/A92) analizado por cromatografía de gases, acreditado por la entidad nacional de acreditación (ENAC) en la norma UNE-EN ISO/IEC 17025 para la realización de ensayos en el sector medioambiental.

Para la medición de la velocidad del aire se utilizó un anemómetro electrónico al mismo tiempo que se llevaba la toma de muestras de COVs. Cada muestra fue tomada a 1 metro de la bahía de trabajo cada 5 min, obteniendo un total de 4 muestras por cada taller.

Para calcular la concentración de COVs en los ambientes se tomó la cantidad de miligramos obtenidos de las muestras dividido para el volumen de aire que se tomó con la bomba (0.9 litros por minuto multiplicado por tiempo de muestreo $15 \mathrm{Min}$, obteniendo un volumen de 13.5 litros de aire).

\section{Resultados}

Se identificó Xileno, Tolueno y Benceno en las 12 muestras analizadas de los 3 talleres automotrices como se detalla en la Tabla 4.

Tabla 4. Concentración de COVs en los talleres automotrices.

\begin{tabular}{llllll}
\hline$N^{\mathbf{N}}$ & Categoría de mantenimiento & Taller & $\begin{array}{l}\text { Xileno mg/m3 } \\
\text { VLA-ED } \leq 221 \\
\text { mg/m3 }\end{array}$ & $\begin{array}{l}\text { Tolueno mg/m3 } \\
\text { VLA-ED } \leq 192 \\
\text { mg/m3 }\end{array}$ & $\begin{array}{l}\text { Benceno mg/m3 } \\
\text { VLA-ED } \leq 3,25 \\
\mathrm{mg} / \mathrm{m} 3\end{array}$ \\
\hline 1 & Ambiente General & $\mathrm{A}$ & 0,0592 & 0,9250 & 0,2510 \\
2 & Ambiente General & $\mathrm{B}$ & 0,3620 & 1,2070 & 0,4370 \\
3 & Ambiente General & $\mathrm{C}$ & 0,0148 & 0,0070 & 0 \\
4 & Limpieza de autopartes & $\mathrm{A}$ & 0,3850 & 1,2220 & 0,4510 \\
5 & Limpieza de autopartes & $\mathrm{B}$ & 0,3180 & 1,1550 & 0,3030 \\
6 & Limpieza de autopartes & $\mathrm{C}$ & 0 & 0 & 0,4900 \\
7 & Limpieza de frenos & $\mathrm{A}$ & 0 & 0,7770 & 0,1030 \\
8 & Limpieza de frenos & $\mathrm{B}$ & 0,3850 & 1,2220 & 0,4510 \\
9 & Limpieza de frenos & $\mathrm{C}$ & 0,7500 & 0,4600 & 0 \\
10 & Limpieza del cuerpo de aceleración & $\mathrm{A}$ & 0,3850 & 1,2220 & 0,4510 \\
11 & Limpieza del cuerpo de aceleración & $\mathrm{B}$ & 0 & 0,5770 & 0,2370 \\
12 & Limpieza del cuerpo de aceleración & $\mathrm{C}$ & 0,6800 & 0,1200 & 0 \\
\hline
\end{tabular}

Notas: VLA-ED es el valor límite ambiental de exposición diaria.

Fuente: Informe técnico ambiental de Laboratorios Himalaya. 
Los promedios de velocidad de aire en los talleres fueron; A: 0,4m/s, B: 0,5 m/s y C: 1,91 $\mathrm{m} / \mathrm{s}$. El taller A con una sola puerta de ingreso se encontraba completamente abierto. El taller B dispone igualmente de una sola puerta de ingreso y se encontraba completamente abierta durante la toma de muestras de velocidad de aire, mientras que el taller $\mathrm{C}$ estaba completamente expuesto al flujo de aire debido a su estructura expuesta al ambiente externo. Los tres talleres solo disponen de ventilación natural.

\section{Análisis de Resultados}

Los talleres "A" Y "B" de ambiente cerrado con ventilación natural poseen mayor concentración de COVs en las 4 categorías de mantenimientos realizados, pero no superan los VLA - ED límites permisibles de acuerdo a las normas de exposición laboral a componentes orgánicos volátiles de España 2015. En el taller automotriz “C” se evidencio una concentración promedio de Xileno 0,3612 mg/m3, Tolueno 0,14675 y Benceno 0,1225 mg/m3. En el taller automotriz "B" de ambiente cerrado posee una concentración en promedio de Tolueno 1,0365 $\mathrm{mg} / \mathrm{m} 3$, Benceno $0,314 \mathrm{mg} / \mathrm{m} 3$ y Xileno 0,2072 mg/m3. En el taller automotriz "A" de ambiente cerrado observó concentraciones promedio de Xileno 0,2073 mg/m3, Tolueno 1,0365 mg/m3 y Benceno 0,314 mg/m3.

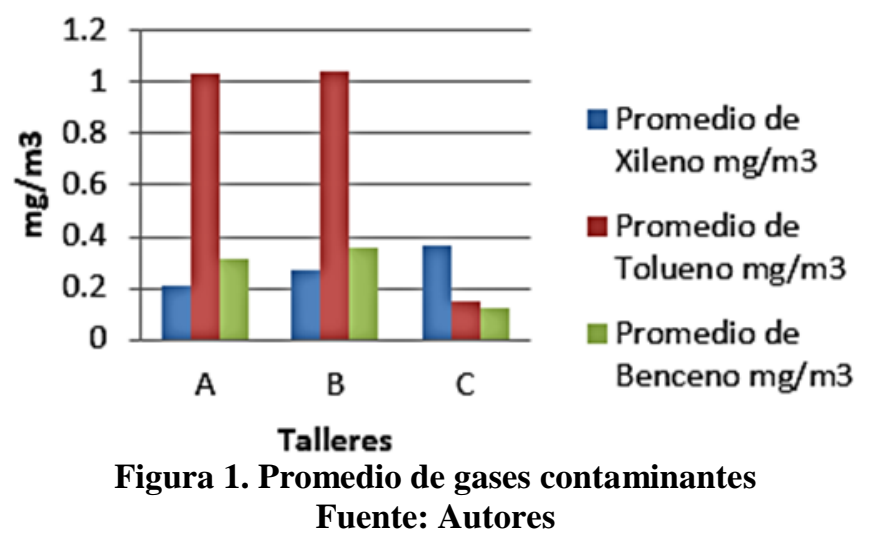

En los talleres automotrices observados se demuestra la presencia de BTX en todos los ambientes laborales automotrices demostrando que el uso de aerosoles provoca concentraciones de Xileno, tolueno y Benceno. Estas concentraciones son afectadas por el tipo de ambiente y el flujo de aire que permite la renovación de aire. Se evidencio que la alta concentración se da en talleres automotrices de ambiente cerrado y a pesar de no poseer una ventilación mecánica en los ambientes cerrados la ventilación natural permitió la renovación de aire. Las menores concentraciones se analizaron en el taller de ambiente abierto influido por el flujo continuo de aire existente.

Estas concentraciones de BTX en los ambientes de talleres automotrices provocan un ambiente incómodo para el desarrollo de las labores de mantenimiento que ademas generan riesgos laborales. La exposición a estos componentes por vías respiratorias, cutáneas y respiratorias causa enfermedades en los trabajadores o ineficiencias al realizar las actividades de mantenimiento (MANZANO, 2015). Durante el estudio y las mediciones realizadas se validó que el uso de aerosoles afecta inmediatamente a la confortabilidad del ambiente y a la salud de 
los trabajadores por lo cual es necesario mantener un uso continuo de equipos de protección personal y usar ventiladores mecánicos para aumentar el flujo de oxigeno dentro del área laboral.

El presente estudio señala que durante los primeros 15 minutos existe una concentración considerable de COVs en las cuatro categorías de mantenimiento por lo cual es necesario un mejor control de ventilación en estas áreas de mantenimiento. En otros estudios se demostró que las exposiciones a estos componentes ocurren en los primeros 1 o 2 minutos inmediatamente después de haber roseado el espray (Wilson, 2007).

\section{Conclusiones}

El análisis de BTX en talleres automotrices de ambiente abierto y cerrado demostró que el uso frecuente de aerosoles limpiadores genera concentraciones bajo los límites permisibles pero afectan a la salud de los trabajadores si no existe un adecuado manejo de estos insumos y el uso continuo de equipos de protección personal además se observó que la ventilación natural a pesar de que permite un flujo de aire no es suficiente para la renovación adecuada del aire.

Los talleres automotrices "A" $\mathrm{y}$ "B" poseen mayores concentraciones de Benceno con un promedio de $0,3770 \mathrm{mg} / \mathrm{m} 3$, Tolueno con $1,1885 \mathrm{mg} / \mathrm{m} 3$ y Xileno con $0,3515 \mathrm{mg} / \mathrm{m} 3$ durante la utilización de aerosoles para la limpieza de autopartes y frenos, sin embargo, estos niveles de concentración no superan los límites permisibles mientras que el taller automotriz "C" posee una menor incidencia de componentes volátiles debido a un mayor flujo de aire.

\section{Bibliografía}

(Insht), I. N. (2015). Límites De Exposición Profesional Para Agentes Químicos En España. 2015. Madrid: Servicios Gráficos Kenaf, S.L.

3117, O. (S.F.). Información Sobre Los Riesgos De Los Productos Químicos. EE.UU.

Agency, E. P. (27 De March De 2016). Federal Register. Recuperado El 28 De 09 De 2016, De Https:/Www.Federalregister.Gov/Documents/2008/03/24/E8-5589/National-VolatileOrganic-Compound-Emission-Standards-For-Aerosol-Coatings

Authority, H. A. (2004). Risk Assessment of Chemical Hazards. Ohio: Noth American Occupational Safety and Health.

Badjagbo, K. (2010). Btex Exposures among Automobile Mechanics. Human and Ecological Risk Assessment, 18.

Chilton. (2000). Manual De Reparacion Y Mantenimiento. United States: Oceano Centrum.

Colaboradores, R. L. (2010). Manual De Seguridad Y Salud Para Operaciones En Talleres Mecánicos Y De Motores Térmicos. Valencia: Universidad Politecnica De Valencia. 
Epa. (17 De March De 2016). Environmental Protection Agency. Obtenido De Https:/Www.Epa.Gov/Indoor-Air-Quality-Iaq/Technical-Overview-Volatile-OrganicCompounds

Industrial, A. C. (2012). Industrial Ventilation Manual. Cincinnati, Ohio: Printed In The United States.

Krzystowczyk, J. A. (2011). Exposure Assessment for Automotive Repair Tasks In. Iowa City, Iowa: University Of Iowa.

Macey, G. P. (2014). Air Concentrations of Volatile Compounds near Oil and Gas Production: A Community-Based Exploratory Study. Journal Environmental Health, 1(13), 82.

Manzano, E. S. (2015). Análisis Toxicológico De Hidrocarburos Aplicado A La Salud Ocupacional. Quito, Ecuador: Pontificia Universidad Catolica Del Ecuador.

Moolla, R. (2015). Occupational Exposure of Diesel Station Workers to Btex. International Journal of Environmental Research and Public Health, 15.

Pérez, A. (2010). Compuestos Volátiles. Recuperado El 01 De 06 De 2016, De Http://Duosalud.Wikispaces.Com/File/View/Productos+Volátiles.Pdf.

Real Decreto 486/1997, España (1997).

Vega, E. J. (2010). Toxicología En Salud Ocupacional. Cuba: Instituto Nacional De Salud De Los Trabajadores.

Weisel, C. P. (2010). Benzene Exposure: An Overview of Monitoring Methods And. Frelinghuysen Road, Piscataway, $\mathrm{Nj}$ 08854, United States: Environmental and Occupational Health Sciences Institute.

Wilson, M. P. (2007). Worker Exposure to Volatile Organic Compounds in the Vehicle Repair Industry. Journal Of Occupational And Environmental Hygiene, 310. 